\title{
Design and evaluation of reliable data transmission protocol in wireless sensor networks
}

\author{
Ailixier Aikebaier ${ }^{\mathrm{a}, *}$, Tomoya Enokido ${ }^{\mathrm{b}}$ and Makoto Takizawa ${ }^{\mathrm{c}}$ \\ ${ }^{a}$ Department of Computers and Systems Engineering, Tokyo Denki University, Ishizaka, Hatoyama, \\ Hiki, Saitama 350-0394, Japan \\ ${ }^{\mathrm{b}}$ Faculty of Bussiness Administration, Rissho University. 4-2-16, Osaki, Shinagawa, Tokyo, 141-8602, \\ Japan \\ ${ }^{\mathrm{c}}$ Department of Computers and Information Science, Seikei University. 3-3-1 Kichijoji-kitamachi, \\ Musashino-shi, Tokyo, 180-8633, Japan
}

\begin{abstract}
A wireless sensor-actuator network (WSAN) is composed of sensor modes and actuator modes which are interconnected in wireless networks. A sensor node collects information on the physical world and sends a sensed value in a wireless network. Another sensor node forwards the sensed value to deliver to an actuator node. A sensor node can deliver messages with sensed values to only nearby nodes due to weak radio. Messages are forwarded by sensor nodes to an actuator node by a type of flooding protocol. A sensor mode senses an event and sends a message with the sensed value. In addition, on receipt of a message with a sensed value from another sensor mode, a sensor node forwards the sensed value. Messages transmitted by sensor nodes might be lost due to noise and collisions. In this paper, we discuss a redundant data transmission (RT) protocol to reliably and efficiently deliver sensed values sensed by sensor nodes to an actuator node. Here, a sensor node sends a message with not only its sensed value but also sensed values received from other sensor nodes. The more number of sensed values are included in a message, the more frequently the message is lost. Each message carries so many number of sensed values that the message loss ratio is not increased. Even if a message with a sensed value $v$ is lost in the wireless network, an actuator node can receive the sensed value $v$ from a message sent by another sensor node. Thus, each sensed value is redundantly carried in multiple messages. The redundancy of a sensed value is in nature increased since the sensed value is broadcast. In order to reduce the redundancy of sensed value, we take a strategy that the farther sensor nodes from an actuator node forward the fewer number of sensed values. We evaluate the RT protocol in terms of loss ratio, redundancy, and delay time of a sensed value. We show that about $80 \%$ of sensed values can be delivered to an actuator node even if $95 \%$ of messages are lost due to noise and collision.
\end{abstract}

Keywords: Wireless sensor-actuator network, WSAN, wireless network, data transmission protocol, reliable data transmission

\section{Introduction}

A wireless sensor-actuator network (WSAN) $[1,2,6,14,15]$ is composed of sensor nodes and actuator nodes which are interconnected in a wireless network. A sensor node $s_{i}$ gathers information on the physical world and sends a sensed value in a wireless channel. The sensor node $s_{i}$ is referred to as initial

\footnotetext{
${ }^{*}$ Corresponding author: Ailixier Aikebaier, 3-3-1 Kichijoji-kitamachi, Musashino-shi, Tokyo 180-8633, Japan. Tel.: +81 42237 3738; E-mail: alisher.akber@computer.org.
} 
sensor node of the sensed value. Messages sent by a source sensor node are forwarded to an actuator node by another sensor node in a type of flooding routing algorithm [6]. There are many discussions on how sensor nodes forward messages to an actuator node so that energy consumption is minimized [6]. On receipt of a sensed value, the actuator node makes a decision on what action to be performed on the real world and performs the action on the real world. A sensor node can deliver a message to another node in its cell. A sensor node is a low-cost, low-energy device with wireless communication facility [5]. Typically, the radius of a cell is about six meters in a Mica Mote sensor node [4,5]. Hence, the farther a destination node is from a source sensor node, the more number of messages sent by the source sensor node are lost by the destination node due to noise in a wireless network. In addition, if multiple sensor nodes whose cells overlap simultaneously send messages, the messages are lost due to collisions. Only simple mechanisms for resolving collisions like the CSMA scheme [9] and TDMA [10] can be used in sensor nodes due to the limited computation power. It is significant to discuss how to reliably and efficiently deliver each sensed value to the actuator node from the source sensor node in present of message loss in the wireless network.

The authors discuss the redundant data transmission (RT) protocol [12,13] to reduce the loss ratio of sensed values sent by sensor nodes even if messages are lost due to noise and collision in a wireless network. If a sensor node $s_{i}$ receives a message sent by another sensor node $s_{j}$, the sensor node $s_{i}$ sends a message with not only a sensed value obtained by $s_{i}$ but also sensed values received from other sensor nodes. Even if a message is lost, the sensed value in the lost message can be carried in other messages. The number of sensed values are carried in a message. The longer the massage length is, the higher possibility the message is lost. A message carries such a limited number of sensed values that the message loss ratio is not increased. It is noted that neither lost messages are retransmitted nor additional messages are transmitted in the RT protocol. Therefore, the number of messages transmitted in a wireless network is not increased in the RT protocol. Since each message can include only a limited number of sensed values, some sensed value may not be forwarded in another message. In addition, since each sensed value is flooding and is carried by multiple messages, the total bandwidth used by sensor nodes are increased. In this paper, we try to reduce the number of messages which carry each sensed value. We take a strategy that the farther sensor node from an actuator node forwards a sensed value with the smaller probability to reduce the number of messages carrying each sensed value.

In this paper, we implement the RT protocol in Mica Mote sensor nodes. We evaluate the RT protocol in terms of the loss ratio, redundancy and delay time of sensed values in presence of message loss. We show the loss ratio of sensed values can be reduced to about $20 \%$ even in an environment where $95 \%$ of messages are lost due to noise and collision in a wireless network. It is noted that the loss ratio of sensed values can be decreased even if lost messages are not retransmitted and any additional control message are not transmitted.

In Section 2, we present the data transmission procedure in the RT protocol. In Section 3, we evaluate the RT protocol in terms of data loss ratio, redundancy, and delivery time.

\section{Redundant data transmission $(\mathrm{RT})$ protocol}

\subsection{Redundant data transmission}

We consider a wireless sensor actuator network (WSAN) where multiple sensor nodes $s_{1}, \cdots, s_{n}$ and one actuator node $a$ which are interconnected in a wireless network. A sensor node $s_{i}$ obtain a sensed value $v$ by sensing an event occurring in an event area of a physical world and sends a message $m$ with 


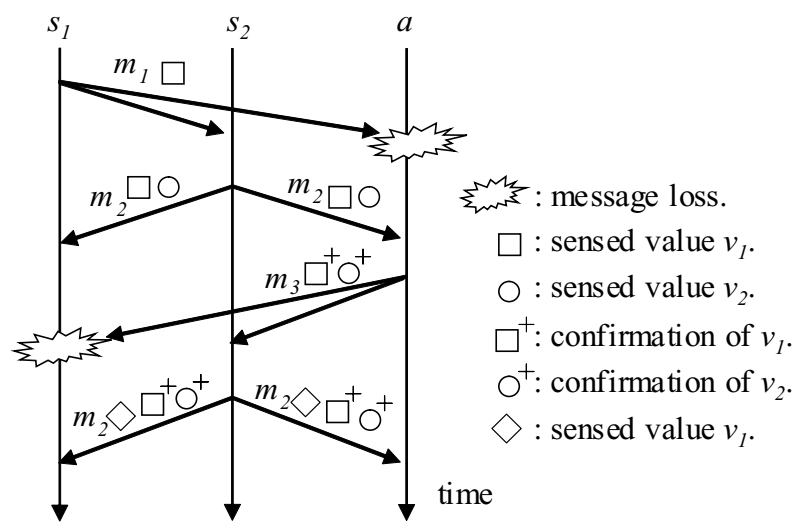

Fig. 1. Message loss.

the sensed value $v$ in a wireless network. A cell is an area where a sensor node $s_{i}$ can deliver a message. Another sensor node $s_{j}$ in the cell of the sensor node $s_{i}$ receives the message $m$ and forwards the message $m$. Thus, a sensed value is forwarded by sensor nodes and delivered to the actuator node $a$.

Suppose the sensor node $s_{1}$ sends a message $m_{1}$ and the sensor node $s_{2}$ receives the message $m_{1}$ but the actuator node $a$ does not receive $m_{1}$ due to noise and collision as shown in Fig. 1. Suppose the sensor node $s_{2}$ sends a message $m_{2}$ after receiving $m_{1}$ and the actuator node $a$ receives $m_{2}$. Here, if the message $m_{2}$ carries a sensed value $v_{1}$ in the message $m_{1}$ in addition to the value $v_{2}$, the actuator node $a$ receives not only the message $m_{2}$ but also the value $v_{1}$ of the lost message $m_{1}$. Data of the message $m_{1}$ carried by the message $m_{2}$ is referred to as backup data of the message $m_{1}$. The message $m_{2}$ is referred to as a backup message of the message $m_{1}$. On receipt of the message $m_{2}$, the actuator node $a$ sends a confirmation message $m_{3}$ for the sensed values $v_{1}$ and $v_{2}$. The sensor node $s_{2}$ receives the message $m_{3}$ but the sensor node $s_{3}$ loses the message $m_{3}$. Then, the sensor node $s_{2}$ sends a message $m_{4}$ with a new sensed value $v_{3}$. Here, the message $m_{4}$ carries the receipt confirmation of the sensed values $v_{1}$ and $v_{2}$. The sensor node $s_{1}$ receives the message $m_{4}$ and knows that the sensed value $v_{1}$ is confirmed, i.e. the actuator node $a$ receives the sensed value $v_{1}$.

On receipt of a sensed value $v_{j}$ from another sensor node $s_{j}$, each sensor node $s_{i}$ forwards the sensed value $v_{j}$ in a message which the sensor node $s_{i}$ sends. Thus, a sensed value is flooding through sensor nodes in a wireless network. A message can include only a limited number $K$ of sensed values and each sensor node can store only a limited number $M$ of sensed values. We have to reduce the number of sensor nodes which forward each sensed value. We take the following strategy:

1. Suppose a sensor node $s_{i}$ sends a message $m_{i}$ with a sensed value $v_{i}$ and a pair of sensor nodes $s_{j}$ and $s_{k}$ receive the message $m_{i}$.

2. If the sensor node $s_{j}$ is nearer to an actuator node $a$ than the other sensor node $s_{k}$, the sensor node $s_{j}$ has the higher possibility that the sensor node $s_{j}$ forwards the value $v_{j}$ than the sensor node $s_{k}$.

The distance $\delta_{i}$ between the actuator node $a$ and a sensor node $s_{i}$ shows how many sensor nodes a message hops to get to an actuator node $a$ from the sensor node $s_{i}$. The longer the distance $\delta_{i}$ is, the smaller possibility a sensor node $s_{i}$ forwards a receipt value.

On receipt of a sensed value $v$ sensed by a sensor node $s_{i}$, the actuator node $a$ sends a confirmation message $m$ with a tuple $\left\langle s_{i}, v, c t\right\rangle$ where $c t=0$ in the wireless network. If a sensor node $s_{j}$ receives the confirmation data $\left\langle s_{i}, v, c t\right\rangle$, the variable $c t$ is incremented by one if the sensor node $s_{j}$ had not received 
any confirmation of the value $v$. Then, the sensor node $s_{j}$ forwards the confirmation data to other sensor nodes. Each sensor node $s_{i}$ collects the values of the variable $c t$ carried in confirmation messages. The average value of the variable $c t$ shows the distance $\delta_{i}$ of the sensor node $s_{i}$.

\subsection{Message format}

A sensor node $s_{i}$ obtains a sensed value $v$ and sends a message with the sensed value $v$ in a wireless network. A message $m$ sent by a sensor node $s_{i}$ has the following attributes:

$-m . s r c=$ source sensor node $s_{i}$ of the message $m$.

$-m . s e q=$ sequence number of the message $m$.

- $m . v a l=$ value $v$ sensed by the sensor node $s_{i}$.

- m.state $=O N$ if the source sensor node $s_{i}$ knows that the value m.val is received by an actuator node $a$, else $O F F$.

- m.data = backup data $\left\langle\right.$ data $_{1}, \cdots$, data $\left._{K}\right\rangle(K \geqslant 0)$.

- m.data ${ }_{j}=$ backup tuple $\langle$ sid, seq, val, state $\rangle$ where sid is an initial sensor node of the sensed value $v a l$, seq is a sequence number of message which the sensor node sid sends, and state is the state of the message whose sequence number is $\operatorname{seq}(j=1, \cdots, K)$.

- m.dist $=$ distance $\delta_{i}$ of the source sensor node $s_{i}$.

If a sensor node $s_{i}$ sends a message $m$ after sending another message $m_{1}$, the message sequence number $s e q$ is incremented by one, i.e. $m . s e q=m_{1} . s e q+1$. In addition to the sensed value m.val, the message $m$ carries the backup data $m$.data which is composed of sensed values which the sensor node $s_{i}$ has received from other sensor nodes. m.data includes the number $K(\geqslant 0)$ of backup tuples. For each backup tuple $d=\langle$ sid, seq, val, state $\rangle$ in m.data, d.state $=O N$ if the sensor node $s_{i}$ receives the confirmation of the sensed value d.val whose initial sensor node is d.sid.

An actuator node $a$ receives messages with sensed values from sensor nodes. Then, the actuator node $a$ sends the following confirmation message $m$ to sensor nodes:

- m.ack = receipt confirmation $\left\langle m . a c k_{1}, \cdots, m . a c k_{A}\right\rangle(1 \leqslant A \leqslant n)$.

- $m . a c k_{j}=$ confirmation tuple $\langle$ sid, seq $\rangle$ showing that the actuator node $a$ receives every message $m^{\prime}$ sent by a sensor node $\operatorname{sid}$, where $m^{\prime} \cdot s e q \leqslant s e q(j=1, \cdots, A)$.

Suppose a sensor node $s_{i}$ receives a confirmation message $m$ which includes a confirmation tuple $<$ $\left.s_{i}, s e q\right\rangle$. Here, the sensor node $s_{i}$ knows that every message whose sequence number is smaller than or equal to $s e q$ and which the sensor node $s_{i}$ sends is received, i.e. confirmed by the actuator node $a$.

\subsection{Data transmission procedures}

If a sensor node $s_{i}$ had received a message $m_{j}$ from a sensor node $s_{j}$, a tuple $\left\langle m_{j} . s r c, m_{j} . s e q, m_{j} . v a l\right.$, $m_{j}$. state, m.dist $\rangle$ is stored in the receipt queue $R Q_{i}$. A message $m_{j}$ is referred to as confirmed in the receipt queue $R Q_{i}$ and attributes $m_{j}$.state of the message $m_{j}$ is changed with $O N$ if the sensor node $s_{i}$ perceives the message $m_{j}$ to be received by the actuator node $a$. A sensor node $s_{i}$ knows that a sensed value $v$ is confirmed not only by receiving a confirmation message of the sensed value $v$ from the actuator node $a$ but also a message from another sensor node which includes the confirmation information that the value $v$ is confirmed. A sensor node $s_{i}$ manipulates a variable $S E Q$ whose initial value is zero. A variable $D_{i}$ indicates the distance $\delta_{i}$ between the sensor node $s_{i}$ and the actuator node $a$. A sensor node $s_{i}$ sends a message $m$ with the sensed value $v$ as follows: 


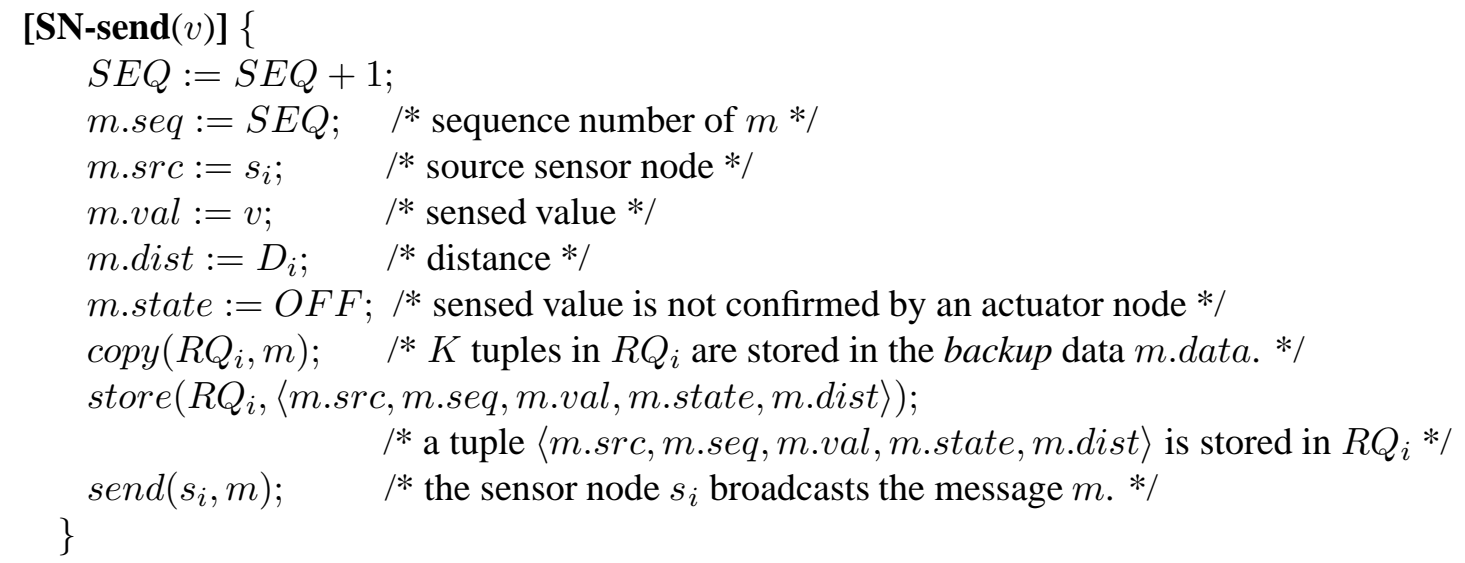

In the procedure copy $\left(R Q_{i}, m\right)$, the top $K$ of backup tuples in the receipt queue $R Q_{i}$ are copied into the backup data of a message $m$. In the procedure store $\left(R Q_{i}, t\right)$, a tuple $t$ is stored in the recept queue $R Q_{i}$. The sensor node $s_{i}$ broadcasts a message $m$ in the procedure $\operatorname{send}\left(s_{i}, m\right)$ in a wireless network.

A sensor node $s_{i}$ forwards sensed values of the receipt queue $R Q_{i}$ in a message $m^{\prime}$ when the sensor node $s_{i}$ sends the message $m^{\prime}$ by the procedure $\mathbf{S N}$-send. In order to reduce the number of messages which carry the sensed values, the sensor node $s_{i}$ does not always store the sensed values in the received message $m$. In this paper, a sensor node $s_{i}$ decides if the sensor node $s_{i}$ stores each sensed value of the message $m$ in the receipt queue $R Q_{i}$ by using the following function:

$$
\delta\left(D_{i}, D_{j}\right)= \begin{cases}1 & \text { if } D_{j}-D_{i} \geqslant 1 . \\ 1 & \text { if } D_{j}=D_{i} \text { and } \operatorname{rand}(1)=0 . \\ 1 & \text { if } D_{i}>D_{j} \text { and } \operatorname{rand}\left(\left\lceil D_{i}-D_{j}+1\right\rceil\right)=0 . \\ 0 & \text { if } D_{i}>D_{j} \text { and } \operatorname{rand}\left(\left\lceil D_{i}-D_{j}+1\right\rceil\right) \neq 0 .\end{cases}
$$

Here, the function $\operatorname{rand}(x)$ gives a random number from 0 to $2^{x}-1$. If a sensor node $s_{i}$ receives a sensed value $v$ whose initial sensor node is $s_{j}$, the sensor node $s_{i}$ stores the sensed value $v$ in the receipt queue $R Q_{i}$ only if $\delta\left(D_{i}, D_{j}\right)=1$ where $D_{i}$ and $D_{j}$ are distances $\delta_{i}$ and $\delta_{j}$ of the sensor nodes $s_{i}$ and $s_{j}$ from the actuator node $a$, respectively.

A sensor node $s_{i}$ receives a message $m$ from another sensor node $s_{j}$ as follows:

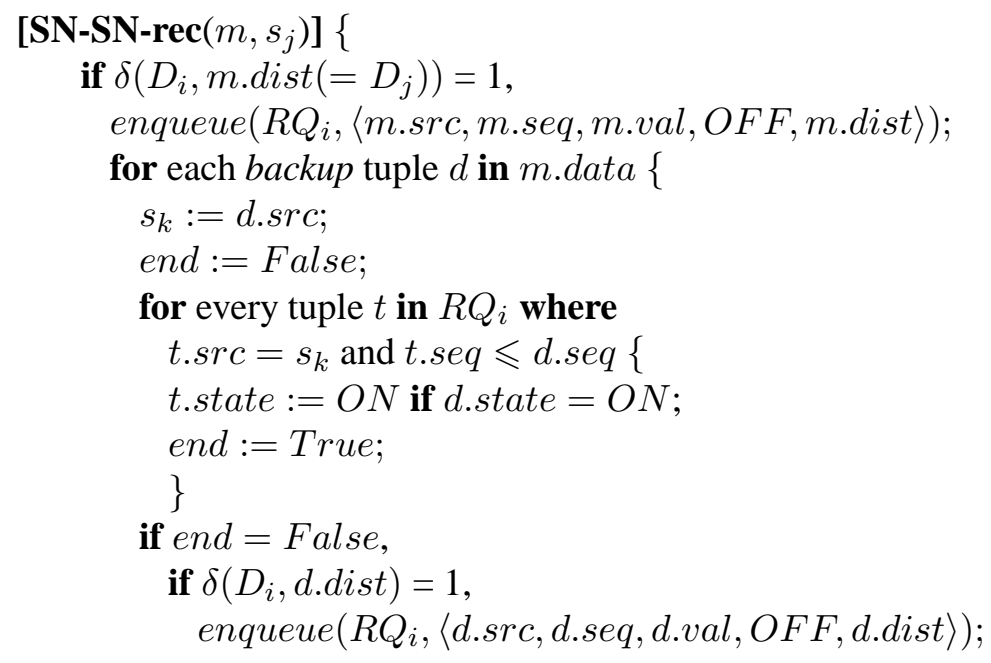




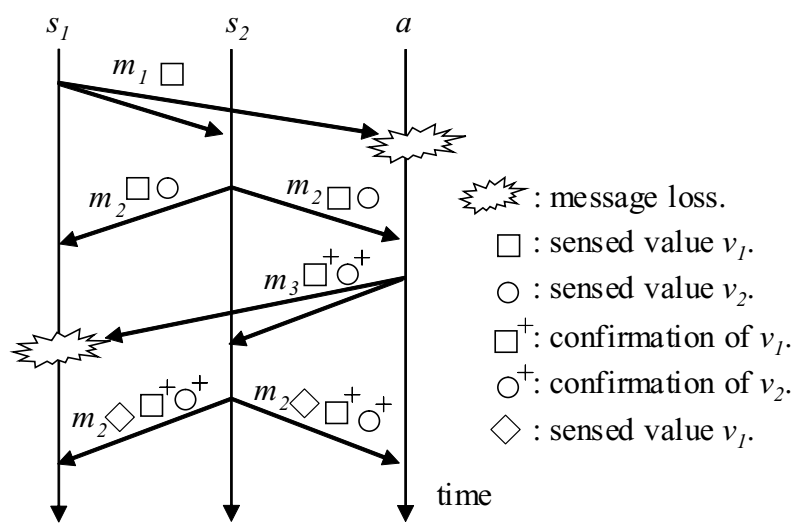

Fig. 2. Forwarding values.

\}

Suppose that a sensor node $s_{i}$ receives a message $m$ from another sensor node $s_{j}$. If the sensor node $s_{j}$ is one hop farther from the actuator node $a$ from the sensor node $s_{i}$, the sensor node $s_{i}$ forwards the sensed value m.val by broadcasting a message including the value m.val. If the sensor node $s_{j}$ is at the same distance as the sensor node $s_{i}$, the sensor node $s_{i}$ discards the value $m$.val with probability $1 / 2$. If the sensor node $s_{j}$ is one hop nearer to the actuator node $a$, the sensor node $s_{i}$ discards the value m.val with probability $1 / 4$. A fewer number of message are sent out to sensor nodes further from the actuator node $a$.

The receipt queue $R Q_{i}$ can include at most $M$ tuples. If the receipt queue $R Q_{i}$ is full, one tuple is removed from the receipt queue $R Q_{i}$ to make a space to store a new tuple. Here, a confirmed tuple is selected and removed in the receipt queue $R Q_{i}$. If the receipt queue $R Q_{i}$ is still full, the top, i.e. oldest tuple of the receipt queue $R Q_{i}$ is removed.

On receipt of sensed values, an actuator node $a$ sends confirmation messages of the sensed values to sensor nodes. If a sensor node $s_{i}$ receives a confirmation message $m$ from the actuator node $a$, the following receipt procedure $\mathbf{S N}$-AC-rec is performed in the sensor node $s_{i}$ :

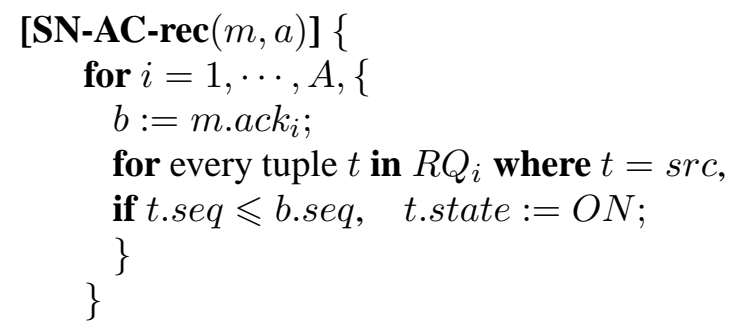

There are the variables $S E Q_{i}, V_{i}$, and $S_{i}$, for each sensor node $s_{i}$ to send a confirmation message in the actuator node $a(i=1, \cdots, m)$. The variable $S E Q_{i}$ shows sequence number of a message which the actuator node $a$ expects to receive next from a sensor node $s_{i}$. The variable $V_{i}$ holds a value sensed by the sensor node $s_{i}$. The variable $S_{i}$ is $O N$ if the actuator node $a$ had sent the receipt confirmation for the sensed value in the variable $V_{i}$ to the sensor node $s_{i}$. Otherwise, the variable $S_{i}$ is $O F F$.

An actuator node $a$ receives a message $m$ from a sensor node $s_{i}$ as follows: 


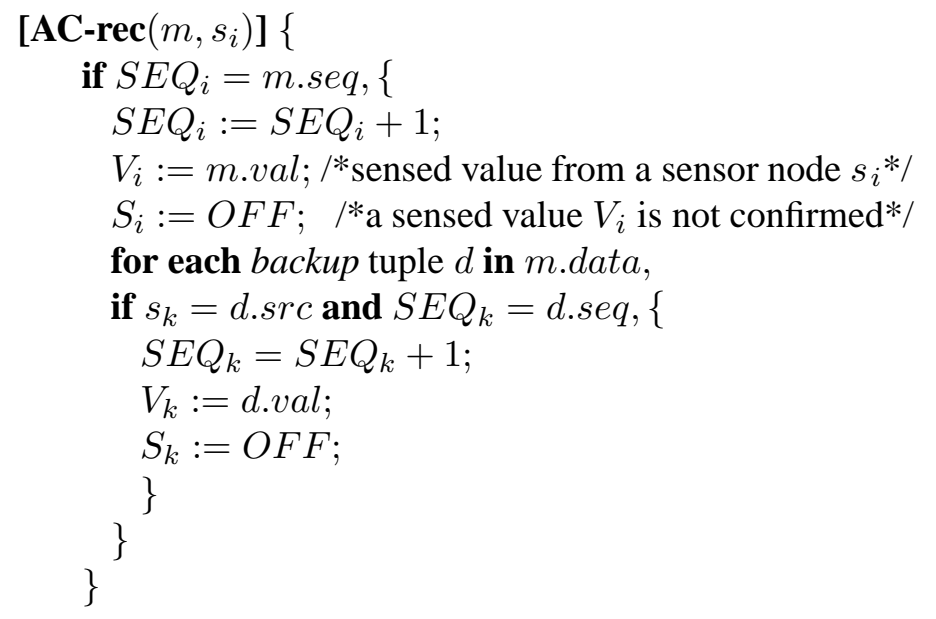

Sensed values from sensor nodes $s_{1}, \cdots, s_{n}$ are stored in a tuple $V=\left\langle V_{1}, \cdots, V_{n}\right\rangle$. An actuator node $a$ sends a confirmation message $m$ of sensed values in the tuple $V$ to sensor nodes.

\section{Evaluation}

\subsection{Assumptions}

In the RT protocol, a message $m$ sent by a sensor node $s_{i}$ carries not only its sensed value $m . v a l$ but also to backup data m.data, i.e. sensed values in another message $m^{\prime}$ which the sensor node $s_{i}$ has received before sending the message $m$. Even if a sensor node $s_{j}$ loses a message $m^{\prime}$, the sensor node $s_{j}$ can receive sensed values in the lost message $m^{\prime}$ if the sensor node $s_{j}$ receives a backup message $m$ of the last message $m^{\prime}$ from the sensor node $s_{i}$. Hence, it is significant to measure how much sensed values are lost. The data loss ratio $(D L)$ is defined to be a ratio of the number of sensed values which an actuator node $a$ loses to the total number of sensed values which the initial sensor nodes send. In traditional protocols, the data loss ratio $D L$ is equal to the message loss ratio. In the RT protocol, the data loss ratio $D L$ is smaller than the message loss ratio. Each sensed value is transmitted in multiple messages. The data redundancy $(R D)$ of a sensed value $v$ is defined to show how many times a message including the sensed value $v$ is transmitted. The data redundancy $R D$ shows how much bandwidth is spent to forward sensed values. Another point is how long it takes to deliver a sensed value to an actuator node $a$. We measure the minimum number of hops $(m H)$ which a sensed value takes to deliver to the actuator node $a$. We evaluate the RT protocol in terms of data loss ratio $(D L)$, data redundancy $(R D)$, and minimum hop number $(m H)$.

We make the following assumptions on the evaluation:

1. There are $n$ sensor nodes $s_{1}, \cdots, s_{n}(n \geqslant 1)$ and one actuator node $a$. The nodes are located in an $m \times m$ mesh where the actuator node $a$ is in the center. The sensor $s_{1}, \ldots, s_{2}$ are deployed in the $m \times m$ mesh. Figure 3 shows a $5 \times 5$ mesh with 24 sensor nodes. Here, $n=m^{2}-1$ and $m$ is an odd number. In this paper, $n=24$ and 48 for $m=5$ and 7, respectively. Let $f$ be the mesh size [m]. Here, $0<f \leqslant 60[\mathrm{~m}]$. The nodes are interconnected in a wireless channel. Let $g$ show the mesh interval $g=f /(m-1)$. 


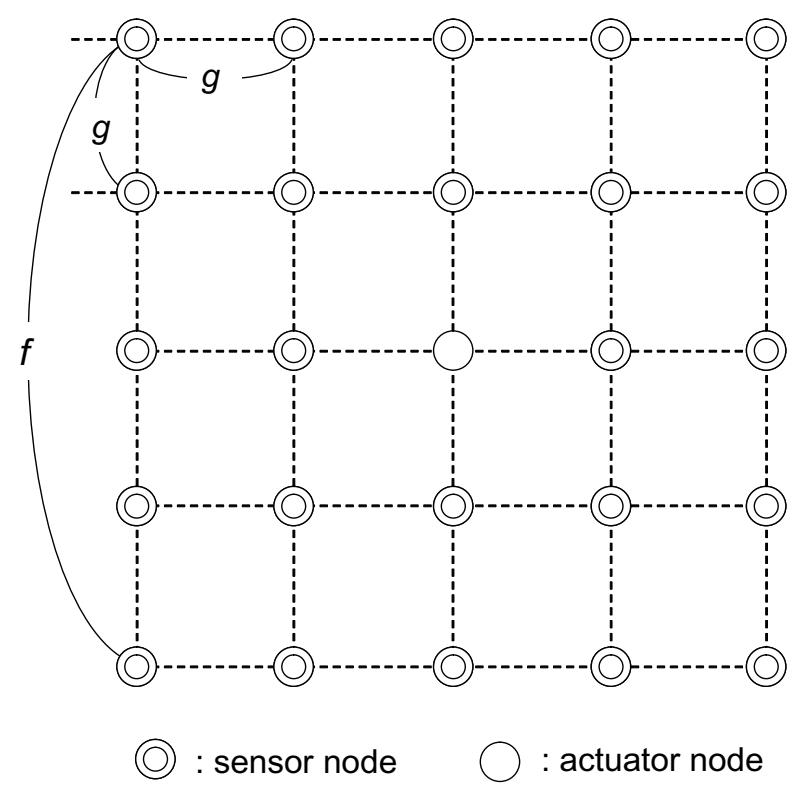

Fig. 3. Mesh structure $(n=24)$.

2. A sensor node $s_{i}$ is realized in a MICA Mote [5]. The MICA Mote is a second generation mote module used for research and development of low power, wireless, sensor networks. The MICA mote was developed by UC Berkeley's research group on wireless sensors. It runs an operating system called Tiny OS. TinyOS is a small, open-source, energy efficient, software operating system developed by UC Berkeley which supports large scale, self-configuring sensor networks.

3. The transmission interval of each sensor node $s_{i}$ follows the normal distribution of average $\tau$ [msec] and variance $\sigma^{2}$, i.e. $N\left(\tau, \sigma^{2}\right)$. Here, $\tau=500$ [msec] and $\sigma^{2}=7500$. Suppose that a sensor node $s_{i}$ sends a message $m_{2}$ after sending a message $m_{1}$. The probabilities that the sensor node $s_{i}$ sends the message $m_{2} 200,500$, and 900 [msec] after the message $m_{1}$ are $0.00114,0.461$, and 0.0000107 , respectively.

4. Each of the sensor nodes $s_{1}, \ldots, s_{n}$ and actuator node $a$ takes the CSMA [9] synchronization scheme against message collision in a wireless channel. Here, each node first listens to the wireless channel before transmitting a message. If the wireless channel is idle, the node start to send the message. In the CSMA/CD scheme $[9,3,16]$, each node still listen to the wireless channel while transmitting the message. If the collision is detected, each node retransmits the message. Since each sensor node is too low-cost, low-power device to realize the CSMA/CD scheme, each node takes the simple synchronization scheme like CSMA.

5. Each message $m$ sent by a sensor node $s_{i}$ includes the number $K$ of backup tuples $(K=$ $0,1, \cdots, M)$. It is noted that " $K=0$ " shows the CSMA protocol, i.e. every message carries just one sensed value and the data loss ratio $(D L)$ means the message loss ratio $(M L)$. " $K \geqslant 1$ " means the RT protocol, i.e. each message carries some sensed value in addition to its sensed value. The size of a sensed value ( $\mathrm{val}$ ) is two bytes long and the sensor identifier $(i d)$ is also two bytes long. That is, the length of one backup tuple is four bytes. Each sensor message carries its sensed value and additional $K$ backup tuples. Each MICA message [5] has a header of thirteen bytes. Here, the length of a sensor message is $17+4 K$ [byte]. 


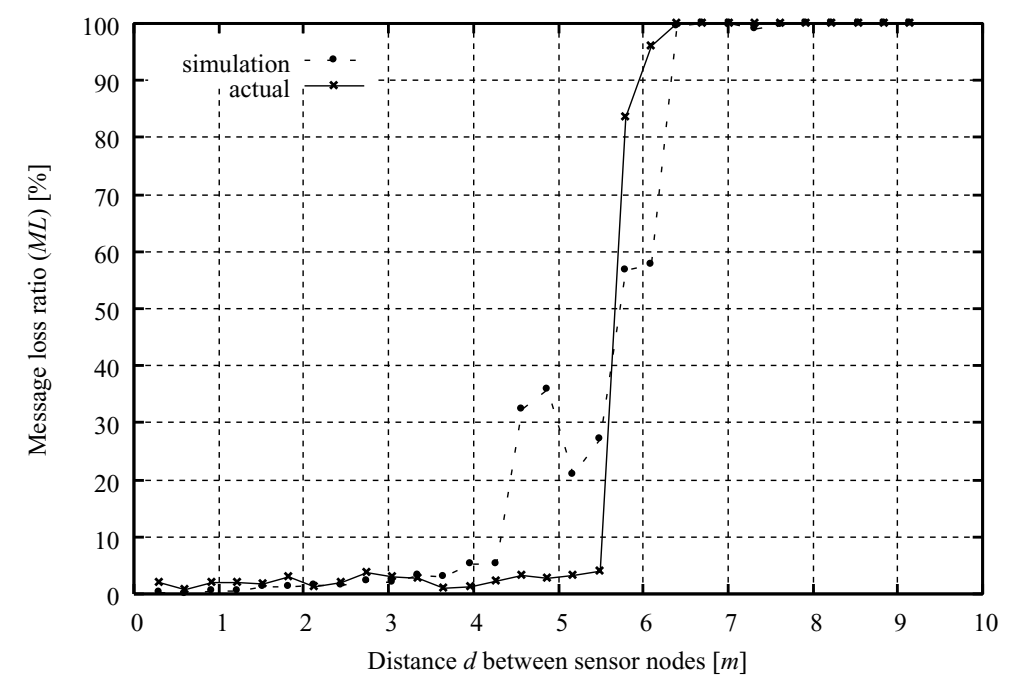

Fig. 4. Message loss ratio due to noise.

6. Each sensor node $s_{i}$ can buffer at most five backup tuples of sensor messages in the receipt queue $R Q_{i}, M=5$. On arrival of a message $m$, the oldest tuple is removed in the receipt queue $R Q_{i}$ to store the information of the message $m$ if the receipt queue $R Q_{i}$ is full.

The RT protocol is realized in the nesC language [7] on the TinyOS operating system [8] of the Mica Mote sensor node. The RT protocol is simulated in the simulator TOSSIM [11] which takes usage of the CSMA scheme [9] as the basic message transmission protocol of a wireless network.

\subsection{Noise and collision}

First, we measure how many messages are lost with respect to the distance among a pair of Mica Mote sensor nodes [5] due to noise. Suppose a sensor node $s_{i}$ sends a message $m$ to another sensor node $s_{j}$ which is $d[\mathrm{~m}]$ from the source sensor node $s_{i}$ in a wireless network. Figure 4 shows the message loss ratio $(M L)$ for distance $d$, which is measured in actual sensor nodes and in the simulation. If $d<5[\mathrm{~m}]$, 2.0 to $5.0 \%$ of the messages are lost due to noise. If $d \geqslant 5$, the message loss ratio $M L$ is drastically increased.

Secondly, we measure how the message loss ratio $(M L)$ changes for size $K$ of a message. We consider three types of messages whose lengths are $17(K=0), 53(K=9)$, and $101(K=21)$ bytes. Figure 5 shows the message loss ratio $M L$ for inter-node distance $d$ in the simulation. If $d \leqslant 3$, the message loss ratio $M L$ is smaller than $5 \%$ even if about 21 sensed values are included in a message. Here, the message loss ratio $M L$ is independent of $K$, i.e. the length of message. This means that we can include more than one sensed value in a message without increase of message loss ratio $M L$.

\subsection{Minimum hop number}

In the RT protocol, sensed values can be delivered to the actuator node $a$ even if some messages with the sensed values are lost. If the sensor node $s_{1}$ is within five meters of the actuator node $a$, the sensed value $v$ can be directly delivered to the actuator node $a$ as shown in Fig. 5. Here, it takes one hop. Even if a message with the sensed value $v$ is lost by an actuator node $a$, another sensor node $s_{j}$ which receives 


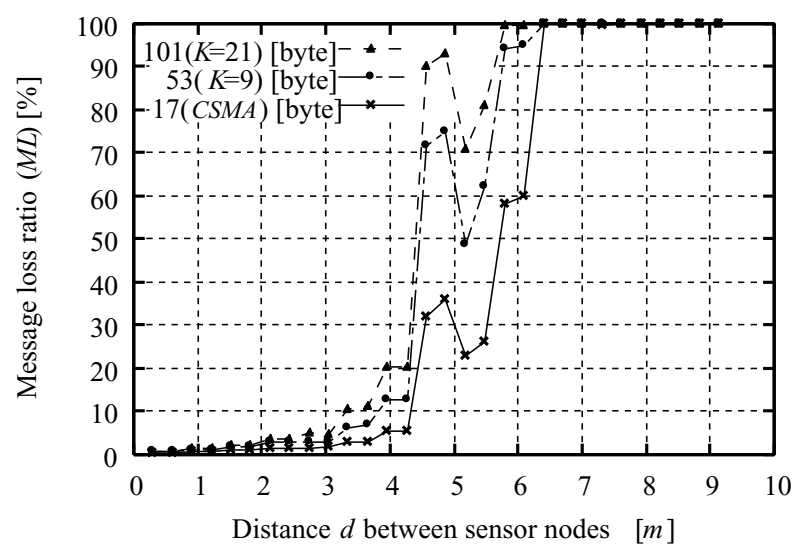

Fig. 5. Message loss ratio with backup tuples.

the sensed value $v$ may forward the sensed value $v$ to the actuator node $a$. Here, it takes two hops. Thus, it takes some number of hops to deliver a sensed value to an actuator node $a$. The larger the mesh interval $g$ is, the more number of hops it takes. An actuator node $a$ thus may receive the sensed value $v$ from multiple sensor nodes. Each instance of the sensed value $v$ takes different number of hops. Here, for each sensed value $v$ which the actuator node a receives, the minimum hop number $(m H)$ is taken out of all the instances of the sensed value $v$ which the actuator node $a$ receives. Here, each sensor node sends messages according to the assumption 2 for 50 seconds. Each sensor node sends 100 messages on average. It is noted that sensed values any of whose instances the actuator node $a$ does not receive are not considered for the minimum hop number $m H$. Figure 6 shows the average minimum hop number $m H$ for mesh size $f(0<f<60)$ where $n=24$, 48. For $K=0$ and $n=24, m H=1$ if $f \leqslant$ 26 [m]. Because every sensed value can be delivered to the actuator node $a$ with one hop. If $K \geqslant 1$, the larger $K$ is, the larger $m H$ is. For example, $m H=2.0,2.5,3.1,3.6$, and 4.2 for $K=1,2,3,4$ and 5, respectively, with $n=24$ and $f=12$ [m]. For example, each sensed value is sent to the actuator node $a$ in two messages on average for $K=1$. In the longer mesh interval $g$, a sensor node can deliver messages to the smaller number of sensor nodes. Hence, the hop number is increased to deliver a sensor node to the actuator node. For example, $m H=1.6,2.0$, and 2.5 with mesh size $f=8,12$, and 20, respectively. For $n=48$, the minimum hop number $m H$ is $1.6,2.5$, and 2.7 with mesh size $f=8,12$, and 20 , respectively. If $f>28$ and $f \geqslant 40$, no message is delivered to the actuator node $a$ since each sensor node $s_{i}$ cannot send messages to any node which is six meters far from the sensor node $s_{i}$ for $n=24$ and 48, respectively, as shown in Fig. 4. Hence, the minimum hop number $m H$ is zero. Figure 7 shows the minimum hop number $m H$ for $K$ where mesh size $f=20$.

\subsection{Data redundancy}

In the RT protocol, a sensed value $v$ sent by the source sensor node is carried in multiple messages. Since a source sensor node $s_{i}$ of each sensed value $v$ sends a message with the sensed value $v$ in a wireless broadcast network, we calculate how many sensor nodes forward the sensed value $v$. The average number of sensor nodes which forward a sensed value $v$ of the sensor node $s_{i}$ is referred to as data redundancy $(R D)$.

First, we do not take any mechanism to reduce the data redundancy. Figure 8 shows the data redundancy $R D$ for the mesh size $f$ where $n=24$ and 48 . Here, each sensor node send messages as explained in 


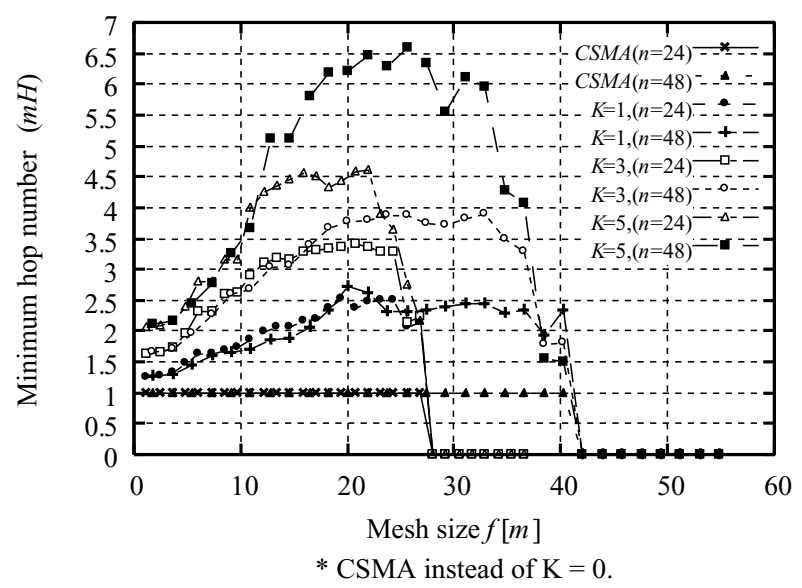

Fig. 6. Minimum hop number $(n=24,48)$.

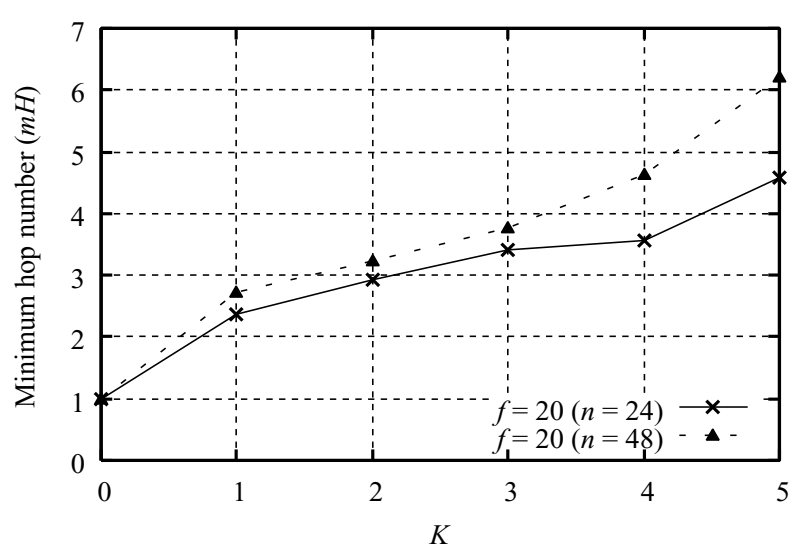

Fig. 7. Minimum hop number vs. $K(n=24,48)$.

the preceding subsection. The larger $K$ is, the higher the data redundancy $R D$ is. For example, the data redundancy $R D$ is $1,2,3,3.8$, and 4.8 for $K=0,1,2,3$, and 4, respectively, with $f=12$ and $n=24$. Three nodes send messages with each sensed value for $K=2$ on average. The longer the mesh size $f$ is, the smaller the data redundancy $R D$ is. The longer the mesh size $f$ is, the more number of messages are lost. Hence, some sensed values are lost since no sensor node receives the messages.

Next, we consider the redundancy reduction (RR) way as discussed in this paper. On receipt of a sensed value $v$ from another sensor node $s_{j-1}$ a sensor node $s_{i}$ forwards the sensed value $v$ with higher possibility if the sensor node $s_{i}$ is nearer to the actuator node $a$ than the sensor node $s_{j}$. Otherwise, the sensor node $s_{i}$ may not forward the sensed value $v$. Figure 9 shows the data redundancy $R D$ with $n=$ 24. For example, about $10 \%$ of data redundancy can be reduced for $K=5$, and $f=20(g=5)$.

\subsection{Data loss ratio}

Finally, we measure the data loss ratio $(D L)$ for $n=24$ and 48. Here, each sensor node $s_{i}$ sends messages according to the assumption 2. The data loss ratio $D L$ for mesh size $f$ is shown in Fig. 10 


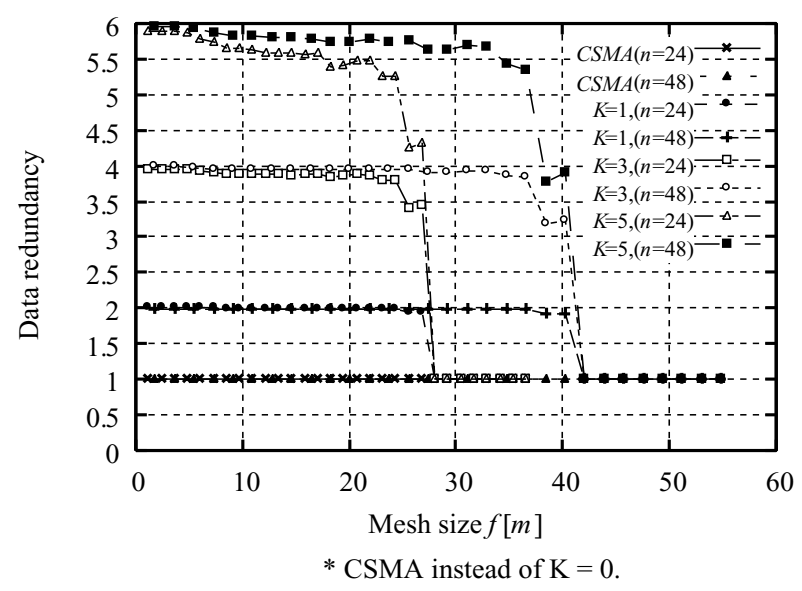

Fig. 8. Redundancy $(n=24,48)$.

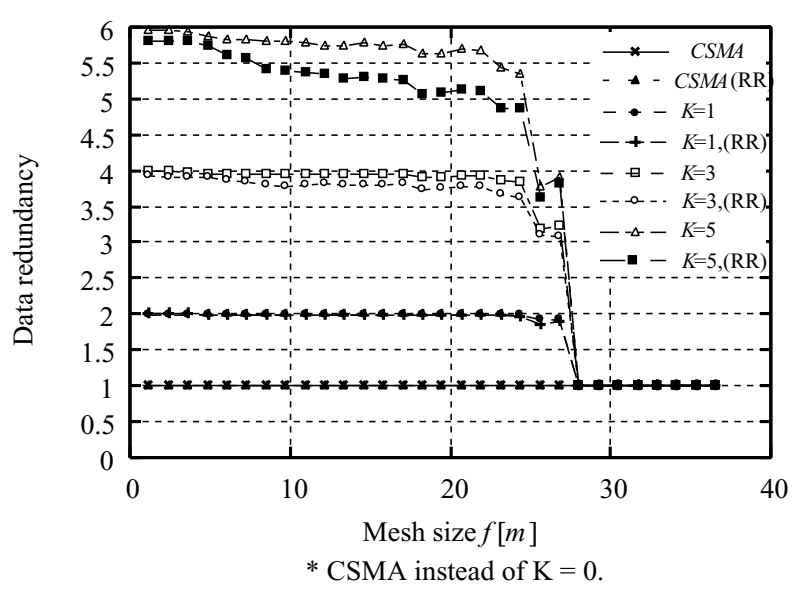

Fig. 9. Redundancy $(n=24)$.

where the redundancy reduction (RR) scheme is not taken. Figures 12-13 show the data loss ratio $D L$ with the redundancy reduction (RR) scheme. Here, each sensor node sends messages as explained in Fig. 6. If mesh interval $g \leqslant 2[\mathrm{~m}]$, the data loss ratio $D L$ is independent of $K$ because every sensor node can deliver a message to the actuator node $a$ with one hop. Here, messages are mainly lost due to collisions, since sensor nodes are closely deployed. Since most messages are lost due to collision, no sensor node receives the messages. Hence, no sensor node can forward sensed values to other sensor nodes. The data loss ratio $D L$ is the same as message loss ratio $(M L)$ and is independent of $K$.

Next, let us consider case the mesh interval $g>2[\mathrm{~m}]$. Some sensor node cannot deliver a message to the actuator node $a$ with one hop. Here, even if some message $m$ is not received by the actuator node $a$, some sensor node might receive the message $m$ and forwards the sensed value in the message $m$ to the actuator node $a$. Hence, the larger $K$ is, the smaller the data loss ratio $D L$ is as shown in Fig. 10. For example, about $72 \%$ and $80 \%$ of the sensed values are delivered to the actuator node $a$ for $K=3$ and $K=4$ although more than $95 \%$ of messages are lost where $n=24$ and $f=24$ [m]. For $K=2$ and 1 , the data loss ratio $D L$ is $35 \%$ and $50 \%$, respectively, with the mesh size $f=24$. In addition, the data 


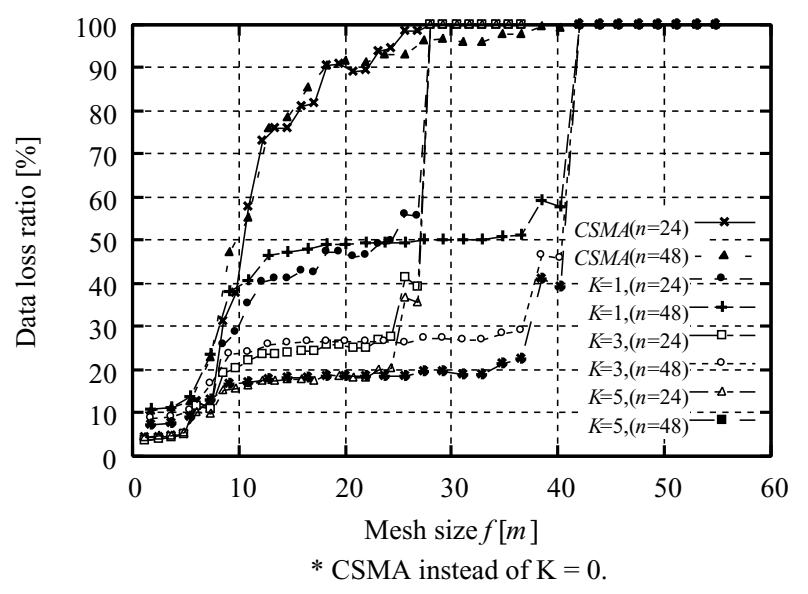

Fig. 10. Data loss ratio $(n=24,48)$.

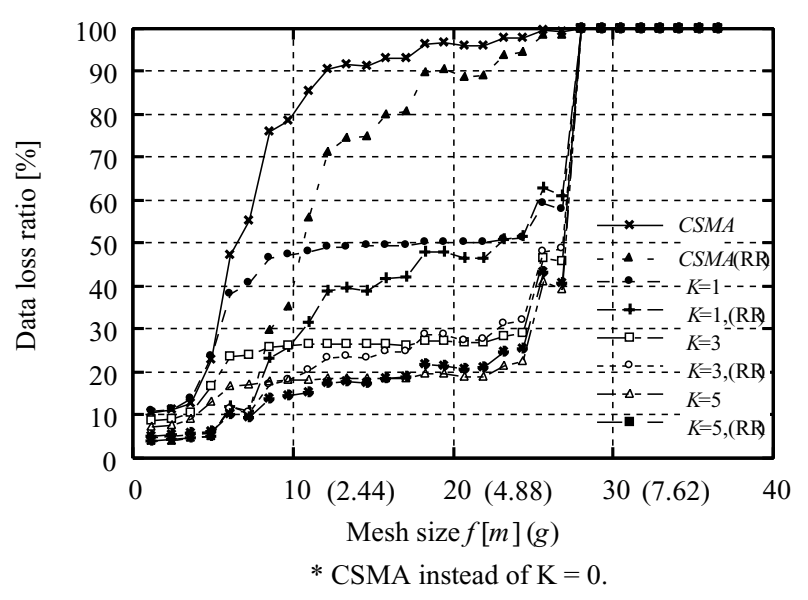

Fig. 11. Data loss ratio ( $n=24, \mathrm{RR})$.

loss ratio $D L$ is increased from $30 \%$ to $100 \%$ and $25 \%$ to $55 \%$ with respect to the mesh size $f, 8[\mathrm{~m}]$ to $28[\mathrm{~m}]$ for $K=0$ and 1, respectively. However, the data loss ratio $D L$ is $25 \%$ to $35 \%$ for $K=2$ and $20 \%$ to $28 \%$ for $K=3$. The data loss ratio $D L$ is not so much increased with $f$ for $K=2$ and 3 as $K=0$ and 1. For $g>7$, i.e. $f>28$ and $f>42$ for $n=24$ and 48, respectively, each sensor node cannot deliver messages to even a nearest sensor node since every pair of sensor nodes are too far. No sensed value is delivered to the actuator node $a$. In the RT protocol with redundancy reduction [RR], the data loss ratio $D L$ can be reduced.

Following Figs 10 to 13, the data loss ratio $(D L)$ can be reduced to $20[\%] \sim 50[\%]$ in the RT protocol. Here, messages are mainly lost due to noise and some sensor node receives a message even if an actuator node $a$ could not receive the message. It is noted the total number of messages transmitted for $K=0,1,2,3$, and 4 is the same. Without increasing the number of messages, the more number of sensed values can be delivered to the actuator node $a$ in the RT protocol. If sensor nodes are very closely deployed, the data loss ratio $D L$ cannot be decreased since most messages are lost due to collision and there is no chance to forward sensed values in another message. The minimum hop number $m H$ shows 


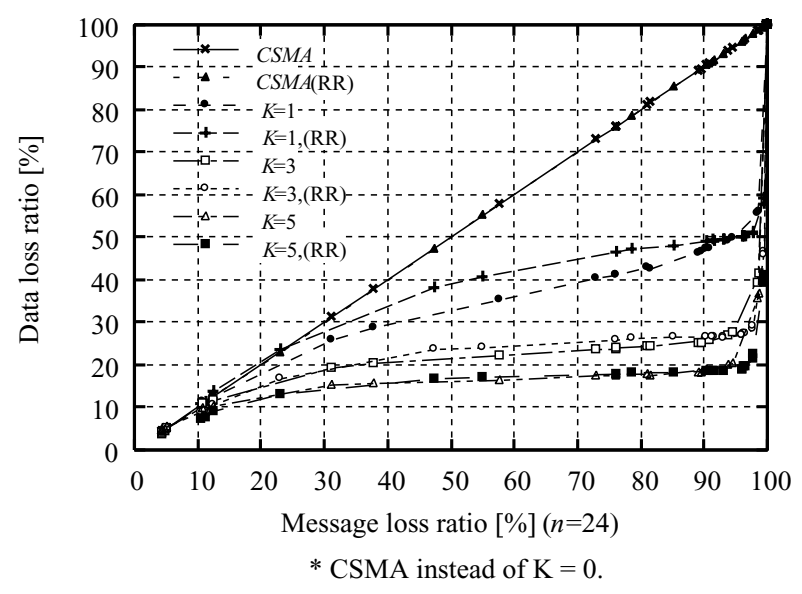

Fig. 12. Data loss ratio vs. message loss ratio.

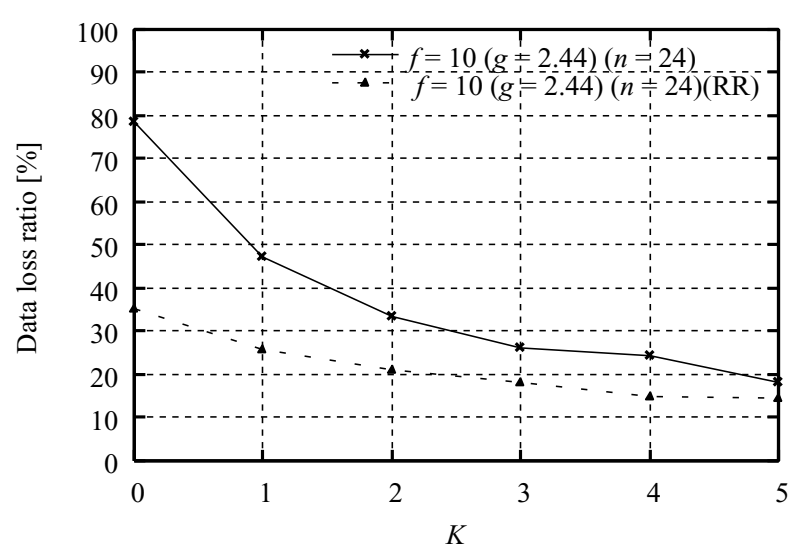

Fig. 13. Data loss ratio vs. $K(n=24)$.

the delivery time of a sensed value. For example, let us consider the mesh size $f$ is 24 [m]. It takes 3.3 hops to deliver a sensed value to the actuator node $a$ and $28 \%$ of sensed values are lost for $K=3$ while it takes 2.5 hops and $50 \%$ of sensed values are lost for $K=1$. For $K=0$, the minimum hop number $m H$ is 1 but $95 \%$ of sensed values are lost. There is thus tradeoff between the minimum hop number $m H$ and the data loss ratio $D L$.

\section{Concluding remarks}

We discussed the redundant data transmission (RT) protocol in a wireless sensor actuator network (WSAN). Messages sent by sensor nodes are lost in a wireless network due to noise and collision. A message received by a sensor node is redundantly forwarded in another message sent by the sensor node in the RT protocol. Hence, even if some messages are lost, an actuator node can receive sensed values in the lost message if the actuator node receives other backup messages carrying the sensed values. It is noted that the number of transmitted messages is not increased in the RT protocol since messages are not 
retransmitted. In addition, we discussed how to reduce the data redundancy of sensed values transmitted by sensor nodes. On receipt of a sensed value $v$ from another sensor node $s_{j}$, a sensor node $s_{i}$ forwards the sensed value $v$ if $s_{i}$ is nearer to the actuator node than $s_{j}$. Otherwise, the sensor node $s_{i}$ may not for ward the value $v$.

We evaluated the RT protocol where sensor nodes are deployed in the mesh structure. In the RT protocol, it is noted lost messages are not retransmitted since sensed values in the lost messages are carried by other messages. We showed that the data loss ratio, i.e. the loss ratio of sensed values can be reduced without retransmission of lost messages in the RT protocol. Only about $20 \%$ of sensed values are lost with $K=4$ even if more than $95 \%$ of messages are lost. In a situation where $50 \%$ of messages are lost, only $22 \%$ of the sensed values are lost with $K=3$.

\section{Acknowledgment}

We would like to thank Mr. Kiyohiro Morita, graduate student of Tokyo Denki University for helping us implement and evaluate the system.

\section{References}

[1] I.F. Akyildiz and I.H. Kasimoglu, Wireless sensor and actor networks: research challenges, Ad Hoc Networks Journal (Elsevier) 2 (2004), 351-367.

[2] I.F. Akyildiz, W. Su, Y. Sankarasubramaniam and E. Cayirci, Wireless sensor networks: a survey, Computer Networks Journal (Elsevier) 38 (2002), 393-422.

[3] Y.-L. Chang and S. Shen, Design of priority schemes in csma/cd local are networks. Proceedings of the 20th annual symposium on Simulation (ANSS'87), 1987, 45-64.

[4] Crossbow Technology, Inc. Wireless sensor networks Getting Started Guide, 2005.

[5] Crossbow Technology, Inc. MPR2400J/420/520 MIB User's Manual, 2006.

[6] A. Durresi and V. Paruchuri. Geometric broadcast protocol for heterogeneous sensor networks. Proc. of 19th IEEE International Conf. on Advanced Information Networking and Applications (AINA2005), 2005, 1:343-348.

[7] D. Gay, P. Levis, R. von Behren, M. Welsh, E. Brewer and D. Culler, The nesc language: A holistic approach to networked embedded systems. Proc. of the ACM SIGPLAN 2003 conference on Programming Language Design and Implementation (PLDI'03), 2003, 1-11.

[8] C.-C. Han, R. Kumar, R. Shea, E. Kohler and M. Srivastava, A dynamic operating system for sensor nodes. Proc. of the 3rd International Conference on Mobile systems, applications, and services, 2005, 163-176.

[9] I. Joe and S.G. Batsell, Reservation csma/ca for multimedia traffic over mobile ad-hoc networks, IEEE International Conference on Communications 3 (2000), 1714-1718.

[10] K.H. Kim, K. Fujiwara, M.-C. Kim, L. Zheng, K. Watanabe and M. Takizawa, A ttf-based programming model and a support kernel running on a communicating sensor platform. Proc. of the 8th International Symposium on Autonomous Decentralized Systems (ISADS2007), 2007, 368-376.

[11] P. Levis, N. Lee, W. Welsh and D. Culler, Tossim: accurate and scalable simulation of entire tinyos applications. Proceedings of the 1st international conference on Embedded networked sensor systems, 2003, 126-137.

[12] K. Morita, K. Ozaki, K. Watanabe, N. Hayashibara, T. Enokido and M. Takizawa, Sensor-actuator communication protocols in wireless networks. Proc. of the First International Conference on Network-based Information Systems (NBiS2007), 2007, 11-19.

[13] K. Morita, K. Watanabe, N. Hayashibara, T. Enokido and M. Takizawa, Evaluation of reliable data transmission protocol in wireless sensor-actuator network. Proc. of the 3rd IEEE International Workshop on Heterogeneous Wireless Sensor Networks (HWISE2007), 2007, 713-718.

[14] V. Paruchuri, A. Durresi and L. Barolli, Energy aware routing protocol for heterogeneous wireless sensor networks. Proc. of 16th International Workshop on Network-Based Information Systems (NBiS2005), 2005, 133-137.

[15] V. Paruchuri, A. Durresi, L. Barolli and M. Takizawa, Three dimensional broadcast protocol for wireless networks. Proc. of the 36th International Conference on Parallel Processing (ICPP2007), 2007, 10-14.

[16] Z. Yao, P. Fan and Z. Cao, An enhanced csma-ca mechanism for multihop ad hoc networks. Communications, 2004 and the 5th International Symposium on Multi-Dimensional Mobile Communications Proceedings, 2004, 2:966-970. 
Ailixier Aikebaier was born in 1981. He received B.E degree in Computers and Systems Engineering from XinJiang University, China, in 2000. He is currently a graduate school student in the Department of Computers and Systems Engineering, Tokyo Denki University. His research interests include distributed systems, P2P network, consensus problems, and fault-tolerant systems.

Tomoya Enokido was born in 1974. He received B.E. and M.E. degrees in Computers and Systems Engineering from Tokyo Denki University, Japan, in 1997 and 1999. After he worked for NTT Data Corporation, he joined Tokyo Denki University in 2002. He received his D.E. degree from Tokyo Denki University in 2003. He is currently a lecturer in Faculty of Business Administration, Rissho University. He won the best paper awards of IEEE AINA 2004 and 2005. He worked for program co-chairs of ICPADS, MNSA2005, and UISW2005. His research interests include distributed systems, group communication, distributed objects, fault-tolerant systems, and distributed transaction management. He is a member of IEEE and IPSJ.

Makoto Takizawa was born in Tokyo, Japan, in 1950. He is currently a professor in the Department of Computer and Information Science, Seikei University since April of 2008. He was a visiting professor at GM-IASI (currently Fraunhofer), Germany (1989-1990) and at Keeled University, England (1990). He is also a visiting professor of Indian University, Xi'an, China since 2004. He joined JAPED (Japan Information Processing Center) in 1975 and developed a heterogeneous distributed system JUDD'S. He joined Tokyo Denki University in 1986. He is a Board of Governors (BOG) from 2003 to 2008 and a Golden Core member of IEEE Computer Society. He is a fellow of Information Processing Society of Japan (IMPS). He was a member of executive board of IMPS from 1999 to 2001 and chaired a SIDES of IMPS from 1997 to 2000. He was a general co-chair and a program co-chair of IEEE ICDCS (International Conference on Distributed Computing Systems) in 2002 and 1998, respectively. He also chairs many international conferences like IEEE ISCOR, MIPADS, and DEXA. He founded IEEE International Conference on Advanced Information Networking and Applications (AINA). He won the best paper awards at ICON-9 (1994), ICON-18 (1998), MIPADS (1996), DAMS (2001), ICPADS2005 (2005), BOCA (2007), and CRISIS(2008), and excellent paper award at AINA-2004. His research interests include distributed systems, group communications, fault-tolerant systems, and information security. 

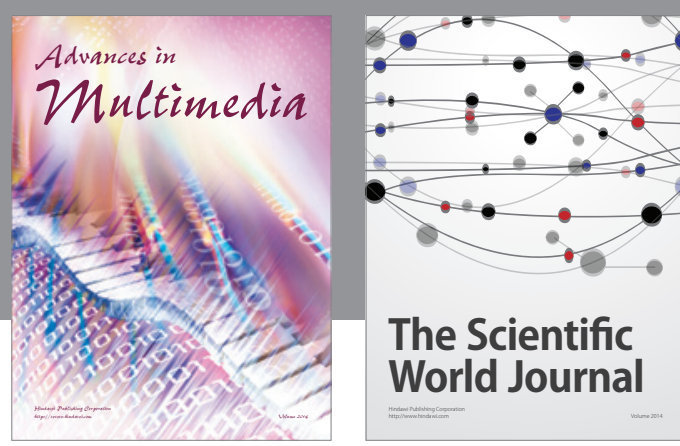

The Scientific World Journal
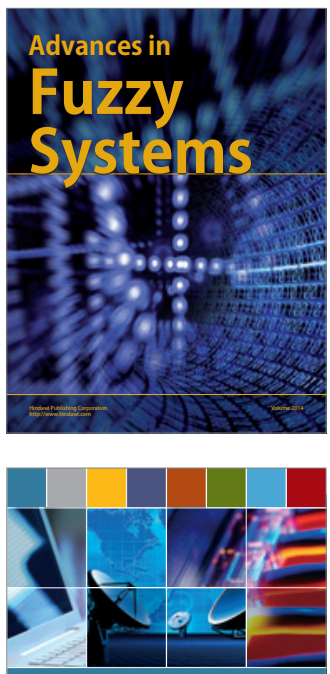

Computer Networks and Communications
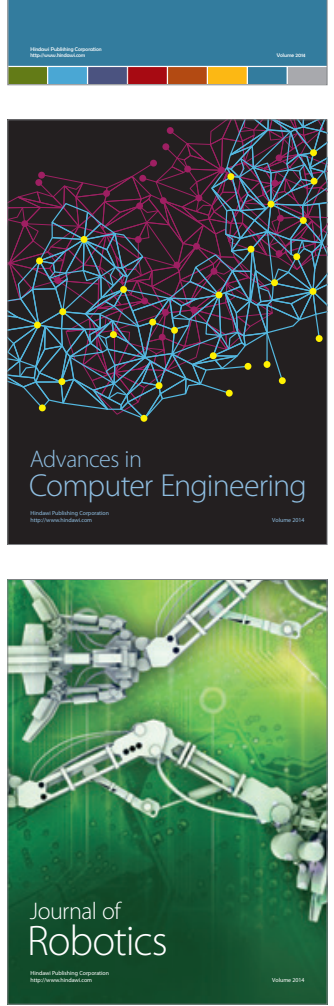
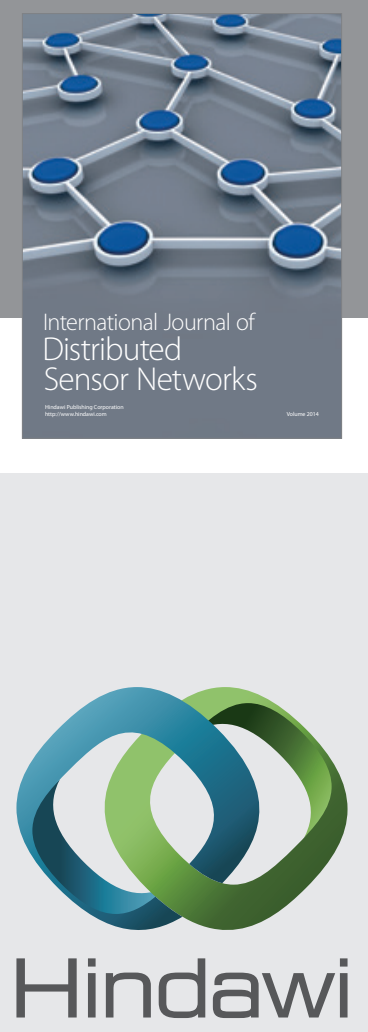

Submit your manuscripts at

http://www.hindawi.com
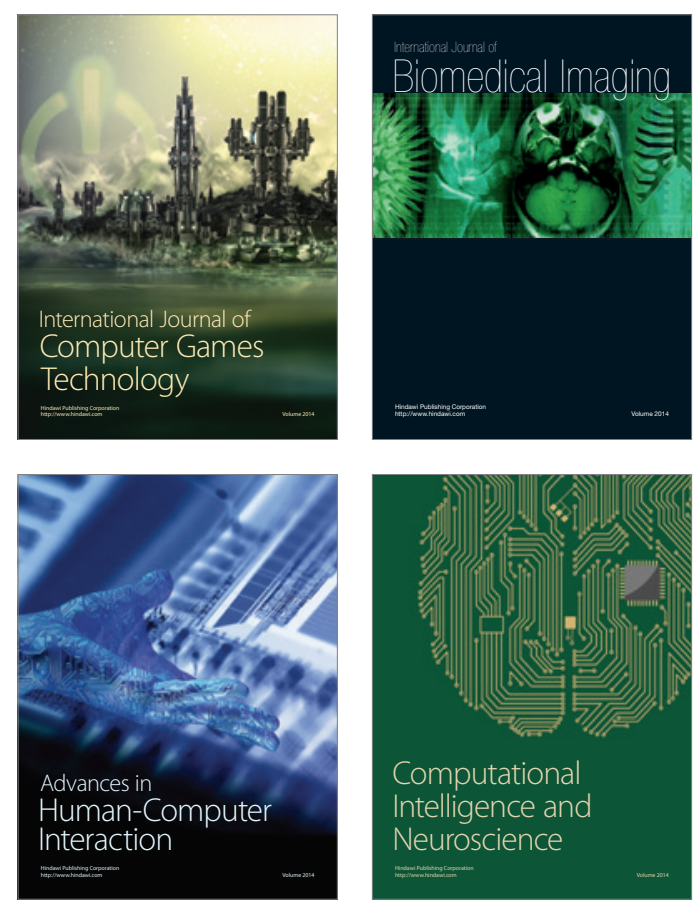
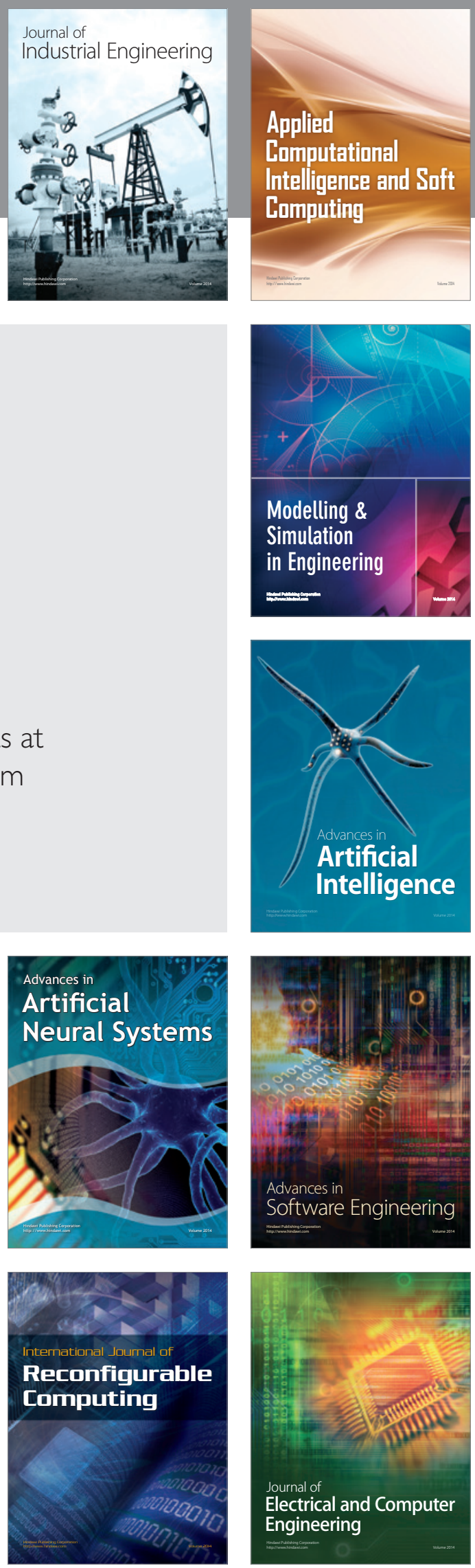